\title{
Updating Student Profiles in Adaptive Mobile Learning using ASP.net MVC, dotNetRDF, Turtle, and the Semantic Web
}

\author{
https://doi.org/10.3991/ijim.v11i3.6165 \\ Dr. Samir E. Hamada \\ Farmingdale State College, Farmingdale NY, USA \\ shamada@bridgeport. edu \\ Dr. Ibrahim Alkore Alshalabi \\ Ma'an Community College, Al-Balqa Applied University, Jordan. \\ ialkorea@my.bridgeport.edu \\ Dr. Khaled Elleithy \\ University of Bridgeport, Bridgeport CT, USA \\ elleithyabridgeport.edu \\ Dr. Ioana Badara \\ University of Bridgeport, Bridgeport CT, USA \\ ibadara@bridgeport.edu \\ Dr. Saeid Moslehpour \\ University of Hartford, Hartford CT, USA \\ moslehpou@hartford.edu
}

\begin{abstract}
In this paper, we present a conceptual framework for using the Semantic Web to get student data from other educational institutions, enabling the educational institutions to communicate and exchange student data. Educational institutions could then use this information to adjust the students' profiles and modify their learning paths. Semantic Web will create a more personalized dynamic course for each student, according to his/her ability, educational level, and experience.
\end{abstract}

Keywords - Adaptive Learning; dotNetRDF; Graphs; mLearning; Ontology; RDF; Semantic Web; Shortest Path; Turtle; User Profile.

\section{Introduction}

Throughout the most recent decades, numerous research studies have examined the possibilities of changing the educational instruction model from the customary onesize-fits-all model to a more adaptive and customized learning model. Most of the techniques calculate the optimal learning path, based on the characteristics in the 
student's profile to make the course more personalized. However, we have not seen any technique that updates profiles dynamically using the Semantic Web to exchange information between educational institutions. The student profile contains information about the student (such as first name, last name, address, course units that have been completed, and grades of those course units). "eLearning is learning utilizing electronic technologies to access educational curriculum outside of a traditional classroom. In most cases, it refers to a course, program, or degree delivered completely online." [1].

This current model can be applied to mobile learning and eLearning in community colleges, as well as, in a typical graduate or undergraduate program at the university level for any course. Students could benefit from and personalize their college experience and graduate earlier by completing their requirements. However, this model does not apply to K-12 students, because they are outside the scope of our research. Adaptive learning is an educational method that aids students in the learning process according to their needs. In addition, adaptive learning assists instructors in conveying course content to their students in a personalized manner based on the students' ability and background. Furthermore, from a developer's point of view, adaptive learning is a technique using computers and other resources to assist in producing a better learning experience. One of the most challenging tasks for adaptive mobile learning is to create an adaptive course. Several researchers have used different techniques in order to make the course adaptive in terms of the course content and units. To the best of our knowledge, there has been no research that attempts to make the course adaptive in terms of previously completed materials by the students at another educational institution by using the Semantic Web to communicate directly with various educational institutions' systems to acquire the students' profiles.

According to our proposed system, the students complete their profile information at the time of the course registration. If there is a claim that the student has successfully completed a course unit at another educational institution, our system will run a query against the Semantic Web files; this will be performed using the SPARQL (SPARQL Protocol and RDF Query Language) [2], where (RDF) the Resource Description Framework, which "is a general-purpose language for representing information in the Web." [3]. For the purposes of our research, we ran the query against Turtle files ("Terse RDF Triple Language, a concrete syntax for RDF" [4]) on another website to simulate the other educational institution. We were able to obtain the students' profiles and grades in that course unit. If the grades fell within the accepted grade range (which is predetermined by each academic institution's subject matter expert), then that course unit will be marked as completed. Otherwise, the student will be presented with a quiz for that course unit. If the student successfully passes the quiz (it is a computerized quiz provided by our system), the course unit will be marked as completed. However, if the student does not pass the quiz, the student must go through the course unit's learning materials and retake the quiz until he or she successfully passes the quiz in that course unit.

When students sign-up and complete their profile information during the sign-up process, they include the completed course units from different educational institutions. The system will then query the Semantic Web files (Turtle) of that institution to 
obtain the student's profile, verify the student's grade, and determine if the student passed the course unit (according to the passing grades imposed by the subject matter expert of each educational institution using our system). If there is no result to ensure that the student has passed the course unit, the student must take a quiz to evaluate his/her knowledge in that course unit. If the student passes the quiz, the course unit will be marked as completed. Otherwise, the student has to go through that course unit's materials and then retake the quiz in that course unit in order to complete it.

In this paper, we propose a technique using ASP.Net MVC, dotNetRDF, Turtle, and Semantic Web to show how we can exchange information between educational institutions in order to update student profiles (in terms of the course units that have been completed) in order to calculate the optimal path for other course units. Student profiles contain information such as student name, completed course units, and grades in each course unit. As a result, students do not have to learn the same course unit more than once. We oftentimes have introductory modules at the beginning of the course, in which we introduce essential notions/concepts assumed to have been learned elsewhere. To counter the common problem of students forgetting previously learned content over time, the system starts by reviewing previously learned concepts and modules; it then teaches students the new required content in order to finish the course.

\section{$2 \quad$ Related Work}

In 2011, Bhatia and Jain [5] showed that the Semantic Web is an augmentation of the current Web, in which data are characterized to empower computers and individuals to work with better coordination. This coordination will help in our research, because we will communicate with various educational institutions in order to verify student claims. In 2013, Hadi et al. [6] stated that the Internet changed the way we collect and deliver information. In their paper, they expressed that the methodology of executing RDF queries against the Semantic Web information requires an exact match between the inquiry structure and the RDF content. They addressed this problem by converting RDF content into a matrix of features and treated queries as classification problems. They effectively built up a working model framework that exhibited the appropriateness of their methodology; this approach might help in our research, as we will use RDF queries against the Semantic Web data.

In 2013, Soualah et al. [7] stated that new technical capacities exist in the area of learning, because of the improvements to mobile phones and wireless technologies. They expressed that mobile learning (m-learning) is a natural extension of eLearning; it has the ability to make learning available on a wide scale, because of the rapid advancements in wireless technologies and the broad utilization of mobile devices. They also stated that learners have different backgrounds and objectives, located in different learning environments (heterogeneity of time, learning time, visual support, ambient noise, etc.). By having more information about the learners, we could adjust the learning strategies to satisfy every learning need. Their approach consisted of two levels: 
1. The semantic level aimed to express semantic characteristics of learning content and learner context.

2. The behavioral level provided users with only the most relevant information.

Their approach made use of learning practices already deployed in eLearning systems and adapted them to m-learning. This idea is fundamental to our current work, since the new technical capacities provide a greater number of possible tools for enhancing learning. In 2014, Grivokostopoulou et al. [8] stated that eLearning framework is turning into a crucial means of educational delivery. Information delivery is one of the fundaments of this current study.

In 2015, Walia et al. [9] stated that the Semantic Web approach to eLearning provides relevant and meaningful information to the learner. Since the human mind develops its own cognitive structure based on personal experiences and background, the mind is usually ambiguous and inconsistent. It is not difficult to learn and secure semantically associated information when the domain of knowledge is huge and wellconnected. In this method of eLearning, the Semantic Web adds the human conceptual representation and the mechanism for using the learner profile and experience. Providing relevant and meaningful information to the learner is fundamental to our research.

As previously mentioned, various related works have contributed to the foundation of our research. The following studies addressed security issues of the Semantic Web that are relevant to our research, (since we have to secure sensitive data). In their research, Kagal et al. [10] concluded that in order to secure the Semantic Web, the following two fundamental parts are required: (1) a semantic strategy that characterizes security necessities and (2) a distributed policy management approach. Furthermore, in the distributed policy management, each entity can determine its own particular strategy for security and privacy. It is essential for Web entities to have the capacity to express their security clearly. In order to achieve this end goal, Kagal et al. utilize a policy language according to a semantic language to markup security information for Web entities. Kagal et al., also, developed two security frameworks: one for distributed environments and one for supply chain management.

In a study by Thuraisingham [11], he provided an overview of the Semantic Web and discussed security issues. Additionally, he stated that security must apply to all of the Semantic Web layers. The security of the Semantic Web should start at the beginning of the project. Also, he concluded that there are situations in which $100 \%$ security should be guaranteed; however, he acknowledged that there are situations that do not require $100 \%$ security. At this stage we have not incorporated any security policy, because it is not within the scope of our research. However, we intend to incorporate a security policy in a later stage of our research.

In 2015, Alshalabi, Hamada, et al. [12, 13] demonstrated the Learning Path Graph (LPG) that is a good representation of online courses in a computer-based usage of an educational framework. This adaptive learning system is displayed as weighted directed graphs, where each course unit is represented by a node on the graph. The Learning Path Graph represents the structure of domain knowledge, learning goals, and all available learning paths as shown in Figure 1. 


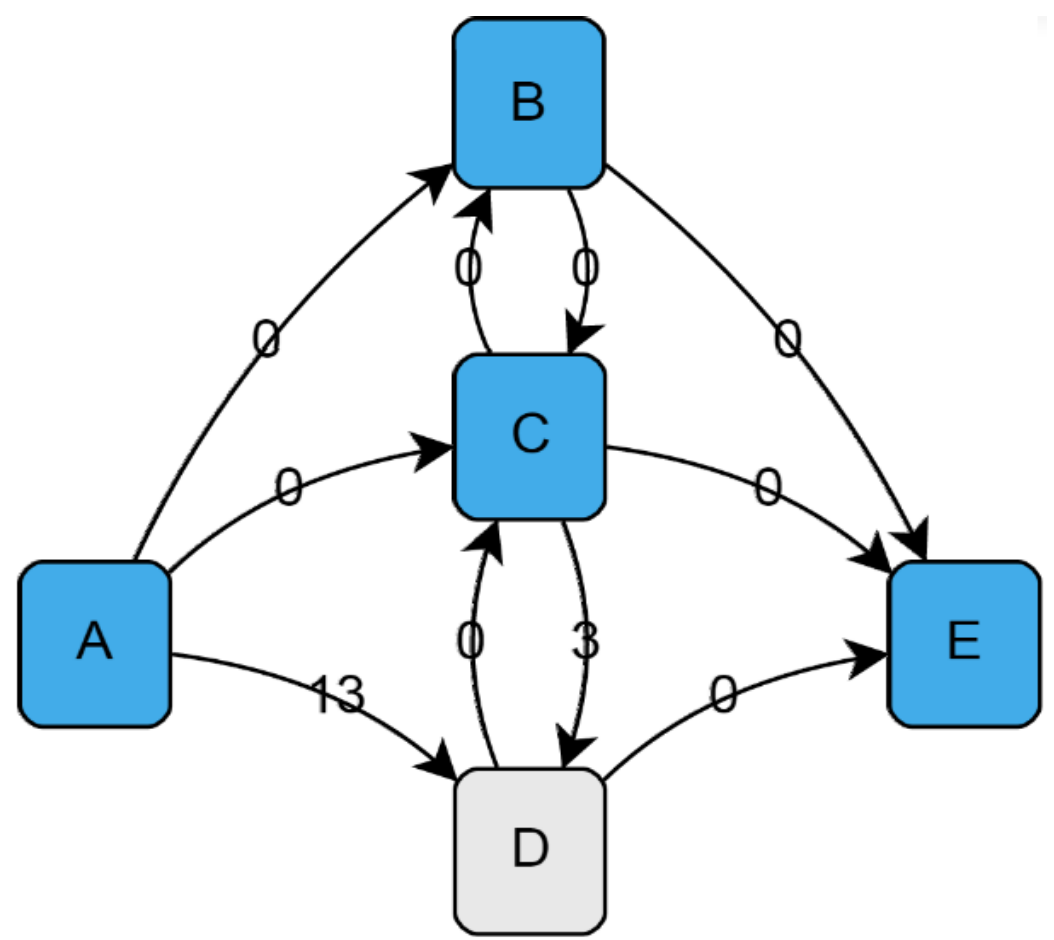

Fig. 1. Learning Path Graph.

In this current research project, we implemented an optimal adaptive learning path algorithm utilizing learner information from the learner profile to enhance specific end goals. This algorithm provides suitable content sequence in a dynamic structure for all learners to accomplish their learning goals in the most effective manner. The optimal path is calculated using our algorithm, which was designed to obtain the lowest cost between the two course units on the path. The cost is determined by the subject matter expert. Cost factors include, but are not limited to, the difficulty level of course units and estimated time required to complete the unit. This study shows how to optimize an adaptive mobile learning system by using the LPG [12]. Furthermore, we will demonstrate how to customize student profiles by using the Semantic Web in order to provide online credit to students for the course units completed in other accredited educational institutions. We, also, describe the conceptual framework of an adaptive mobile learning system and how student profiles are used to adjust the learning path, thereby, making the learning path more dynamic. This means that when students learn a course unit there will be an adjustment to their learning path. A new optimal path will be generated by the system. The interesting point in this study is the ability to use the Semantic Web to exchange student information among educational institutions and to credit students for the course units that they have already completed. This feature may have the potential to boost the efficiency of the adaptive learning systems and increase the chance for student success. 


\section{Proposal}

Ontology, as a term, is derived from the Greek words "onto" (which means being), and the word "logia" (which means written or spoken discourse). Ontology means different things to different people. In philosophy, it represents the study of the existence and nature of being. In the Semantic Web, ontologies are formal definitions or representations of vocabularies or knowledge that allow the user to define resource classes, resource properties, and relationships between resource class members [14, 15]

Eisenstadt and Vincent [16] said that, "An ontology is a partial specification of a conceptual vocabulary to be used for formulating knowledge-level theories about a domain of discourse."

The three parts of the RDF triple are shown below in Figure 2,

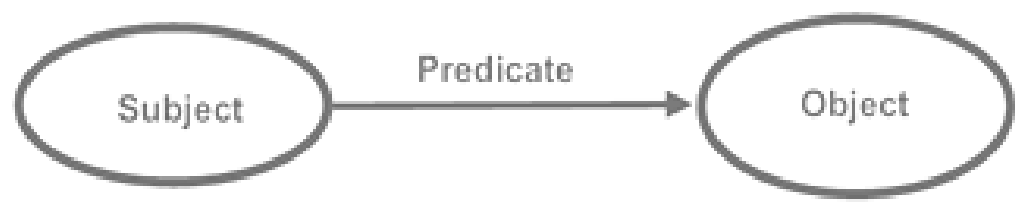

Fig. 2. Resource Description Framework (RDF) Triple.

For example, as shown in Figure 3, the triple "(John) (Knows) (Jane)," (John) is the subject, (Knows) is the predicate, and (Jane) is the object.

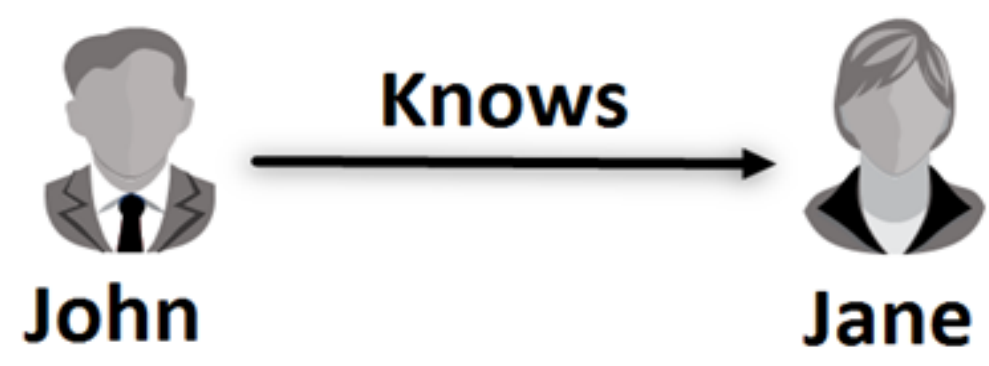

Fig. 3. Resource Description Framework (RDF) Triple Example.

Using Turtle syntax, it can be written as follows as shown in Figure 4.

$$
\begin{aligned}
& \text { @prefix foaf: <http://xmlns.com/foaf/0.1/>. } \\
& \text { foaf:John foaf:Knows foaf:Jane. }
\end{aligned}
$$

Fig. 4. Parts of the Triple in Turtle format.

There are several advantages of ontologies including:

1. Publishing data using common vocabulary and grammar 
2. Preserving data semantic descriptions in ontologies

3. Data are ready for inference

4. Better visibility

5. Extensibility

6. Flexibility

7. Visibility

8. Ability to add new properties at any time without breaking compatibility $[17,18]$.

Table 1 shows a rough interpretation of terms used to describe relational databases and ontologies.

Table 1. Relational Database and Ontology

\begin{tabular}{|l|l|}
\hline \multicolumn{1}{|c|}{ Relational database } & \multicolumn{1}{c|}{ Ontology } \\
\hline row & subject \\
\hline column & predicate \\
\hline table data & literal nodes \\
\hline
\end{tabular}

As shown in Figure 5, we have a students' file in Turtle format and each student has the following properties:

1. ID

2. Given Name

3. Family Name

4. Email

5. Street Address

6. Address Locality

7. Address Region

8. Postal Code

9. Address Country

10. Student Group

The following are some vocabularies from different schemas:

@prefix d: <http://adaptivemobilelearning.com/ns/data\#>

@ prefix person: $<$ http://schema.org/Person $>$

@prefix address: $<$ http://schema.org/>

@prefix place: $<$ http://schema.org/Place/>

@prefix aiiso: <http://purl.org/vocab/aiiso/schema\#>

(a) prefix contains: $<$ http://schema.org/hasPart $>$

@prefix teach: <http://linkedscience.org/teach/ns\#>

@prefix completed: https://schema.org/Completed

With the available schemas listed above, we were able to map to our data files; they might not be an exact match, but this study was about a demonstration of how to be able to get student results from another educational institution using Semantic Web. Figure 6 shows student's data in Turtle format. 


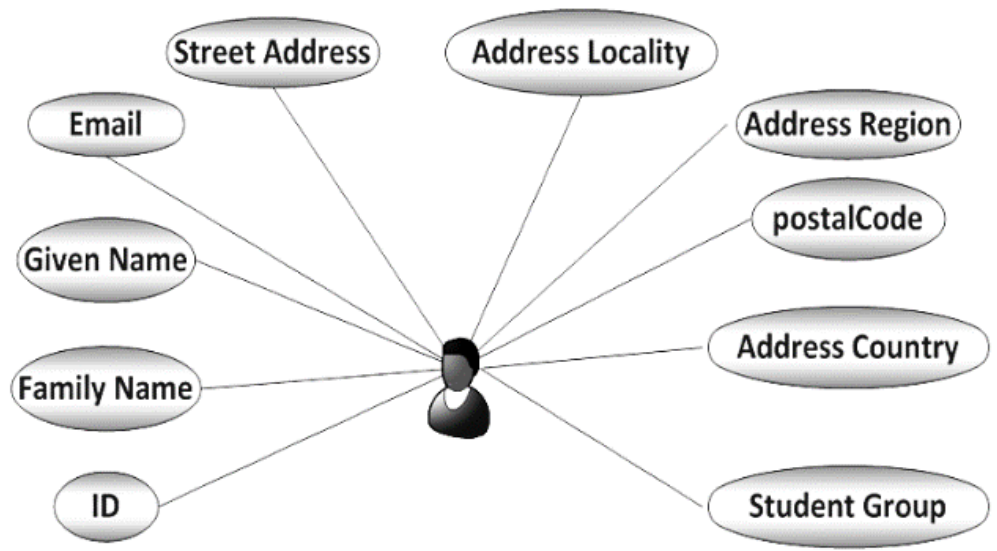

Fig. 5. Student's Properties.

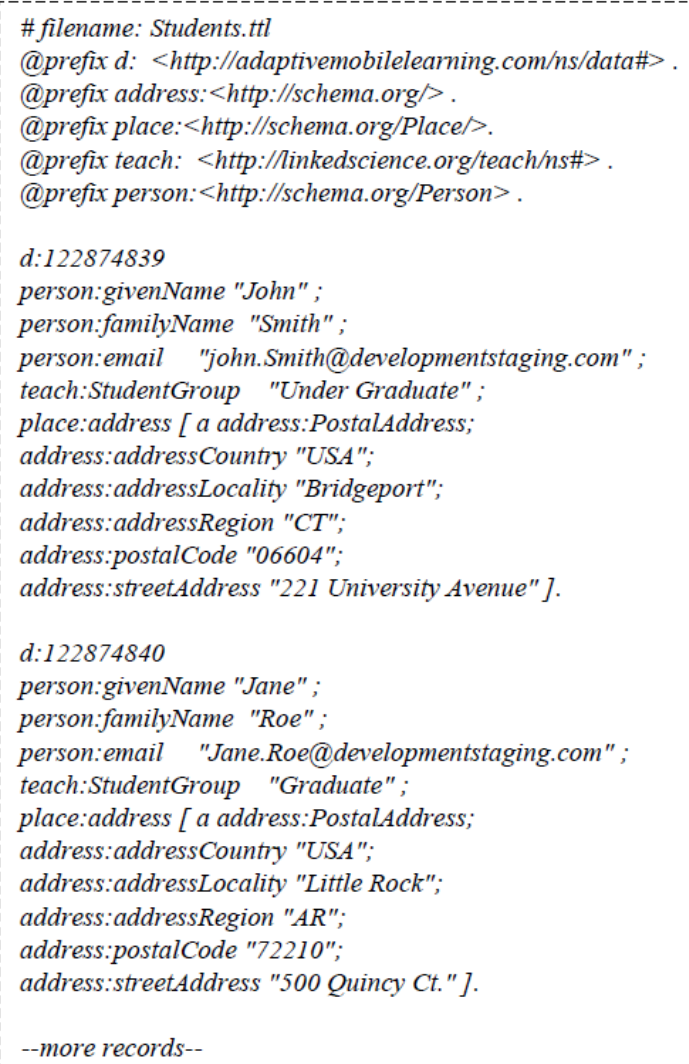

Fig. 6. Students' File in Turtle Format. 
In Figure 7, we are going to use the SPARQL query to select students in Connecticut (CT).

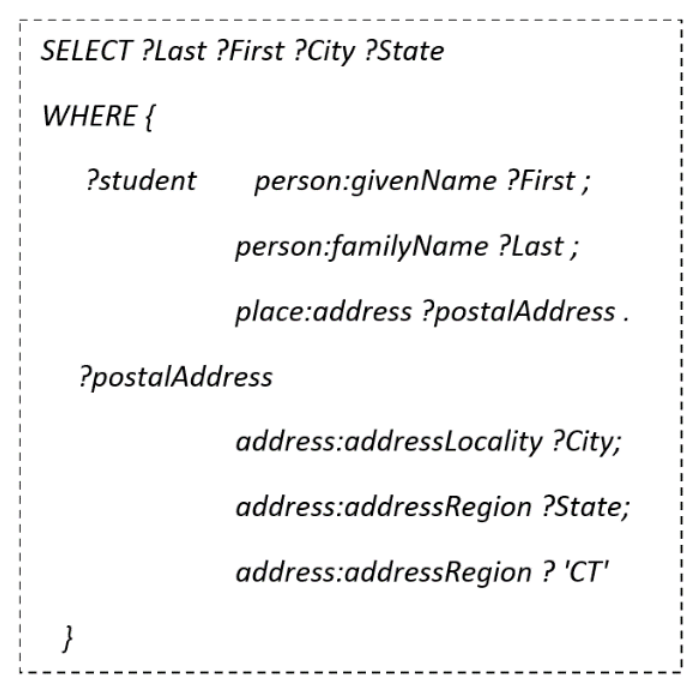

Fig. 7. SPARQL Query for students in CT.

Table 2. Result of query from Figure 7

\begin{tabular}{|l|l|l|l|}
\hline \multicolumn{1}{|c|}{ Last } & \multicolumn{1}{c|}{ First } & \multicolumn{1}{c|}{ City } & \multicolumn{1}{c|}{ State } \\
\hline Smith & John & Bridgeport & CT \\
\hline Bloggs & Joe & Stratford & CT \\
\hline
\end{tabular}

As shown in Table 2, two students are in Connecticut, John Smith and Joe Bloggs. Now, we will add one more condition: the city of Stratford as indicated in Figure 8.

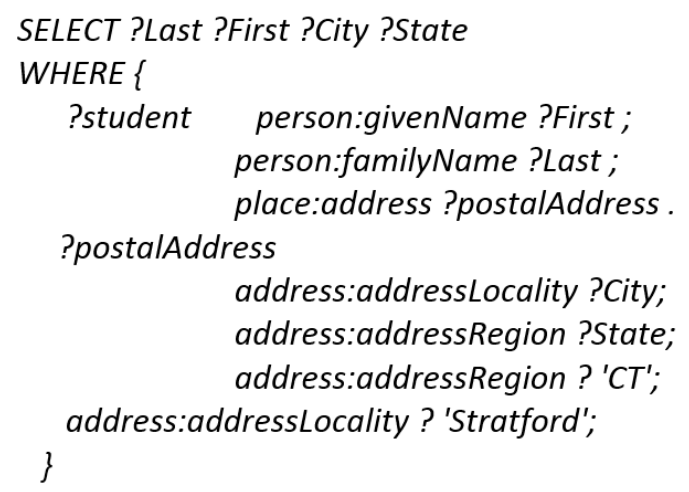

Fig. 8. Query for students in city $=$ Stratford and state $=C T$ 
The only student in our table who lives in Stratford is Joe Bloggs, and the result of running the query confirmed that, as shown in Table 3.

Table 3. Result of query from Figure 8

\begin{tabular}{|c|l|l|l|}
\hline Last & \multicolumn{1}{|c|}{ First } & \multicolumn{1}{c|}{ City } & \multicolumn{1}{c|}{ State } \\
\hline Bloggs & Joe & Stratford & CT \\
\hline
\end{tabular}

Implementing dotNetRDF $[13,14,19]$

The dotNetRDF project aimed to create an open source .Net library using the latest versions of the .Net framework to provide a powerful and easy-to-use API to work with RDF, SPARQL, and the Semantic Web. The primary goal is to provide an efficient method for working with reasonable amounts of RDF in .Net. Using dotNetRDF is extremely simple. Reading Turtle files can be done as follows: The following snippet loads the Turtle files to the computer memory, as a structured graph, as shown in Figure 9.

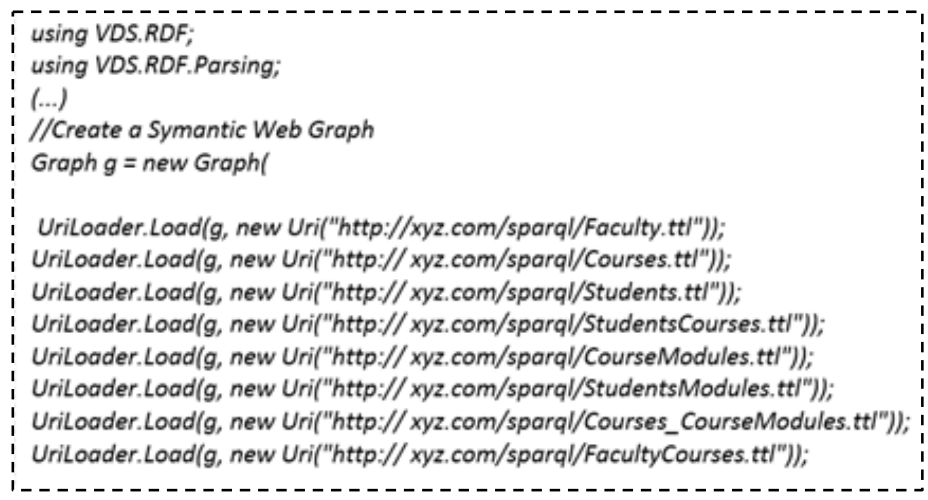

Fig. 9. Loading the Turtle files to memory.

Figure 10 shows the SPARQL query that is going to be executed on graph $\mathrm{g}$.

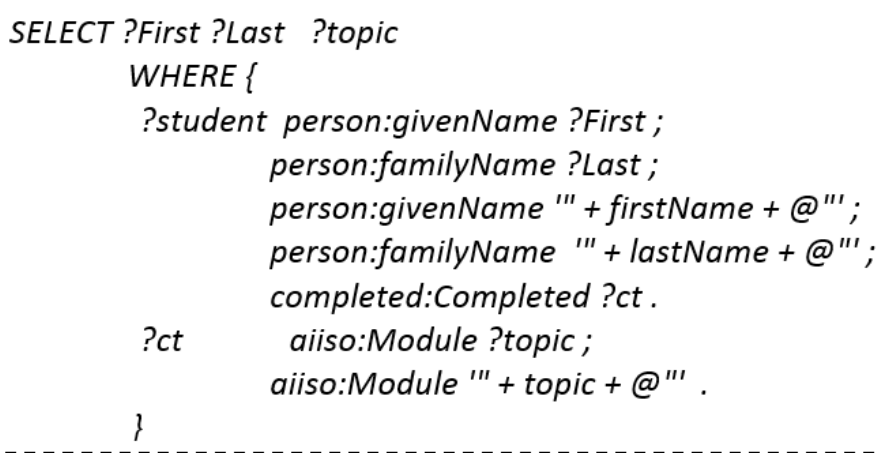

Fig. 10.A SPARQL query on the files loaded in Figure 9 
The value of the variable firstName equals "John", the value of the variable lastName equals "Smith" and value of the variable topic equals "Introduction". The query is going to display last name, first name, and course unit, where first name equals "John", last name equals "Smith", and the course unit equals "Introduction". The SPARQL queries can be executed with ExecuteQuery method, as shown in Figure 11.

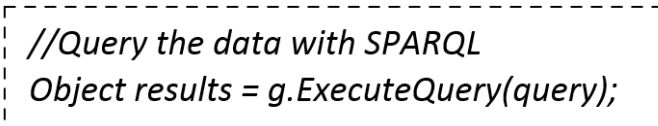

Fig. 11.Executing Query in Figure 10

The ExecuteQuery method runs the query against a loaded ontology. In the following code snippet, we will verify that the query-executed results are not null and then parse them to a SparqlResultSet. The SparqlResultSet consists of a number of SparqlResults. Each SparqlResult corresponds to a single fetched "row". We also will get the count of records and then store it into the ViewData["Count"], which will be displayed in the view. We will then create a ViewData["Result"] in which we will store the value "Passed" if the record count is greater than zero; otherwise we will store "Not Passed." As shown in Figure 12.

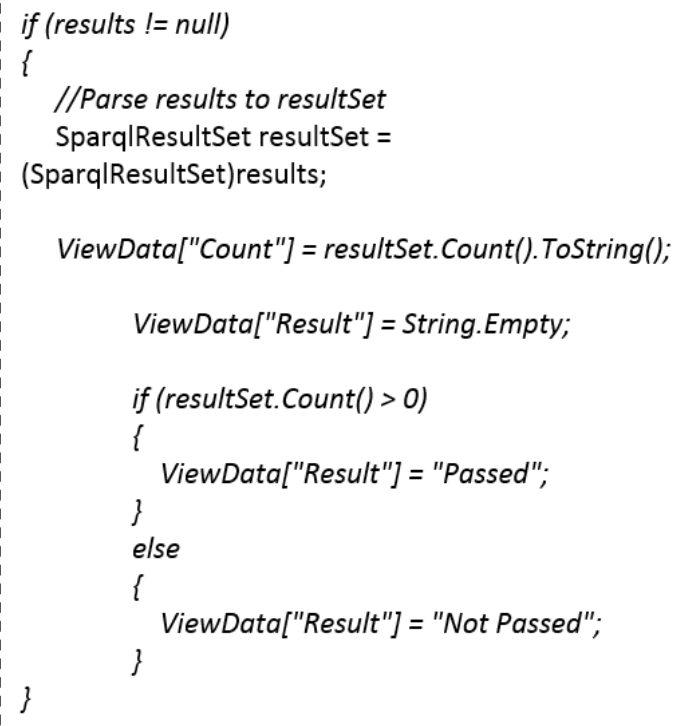

Fig. 12.Evaluating the Query result from Figure 11

\section{Development of the Framework}

We have performed tests on the system using ASP.Net MVC and the Turtle files: 
1. CourseModules.ttl

2. Courses.ttl

3. Courses CourseModules.ttl

4. Faculty.ttl

5. FacultyCourses.ttl

6. Students.ttl

7. StudentsCourses.ttl

8. StudentsModules.ttl

We were able to get student information about the completed course units by providing the parameters, first name, last name, and course unit to the controller via the view. We then received the results that indicated whether or not a student passed the course unit. This information could be used to update the student's profile and used to adjust the learning path to make it more adaptive, according to the system diagram of Figure 13.

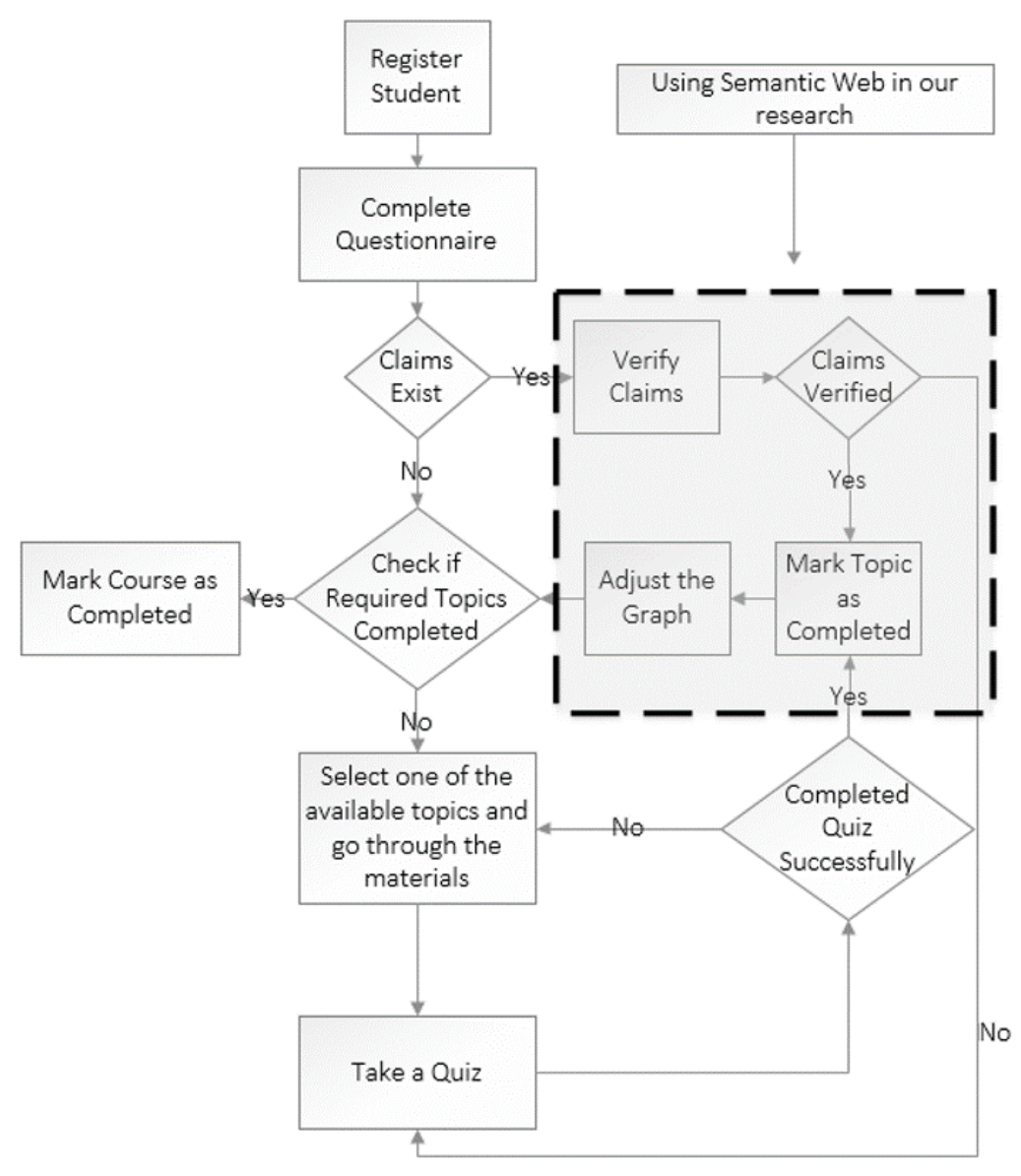

Fig. 13.System Diagram 
Figure 13 shows when a student registers and completes the questionnaire, if there are claims about completing successfully a course unit in another educational institution, the system will query the Turtle files located on that institution's website to verify the claim. Once the claim is verified, the course unit will be marked as completed, and then the system will check to see if the required course units (the units that need to be completed in the course) were successfully completed. The system will then mark the course as completed; otherwise, the student has to complete the course unit quiz successfully in order to mark this course unit as completed. However, if the student does not pass the quiz, then the student must finish one of the available course units and take the quiz. Upon passing the quiz successfully, the course unit will be marked as completed. The system will then check if the required course units to complete this course are successfully completed. The system, afterwards, will mark the course as completed.

Figures 14 through 17 illustrate the relationship between the student and completed course units using the relations between the Turtle files.

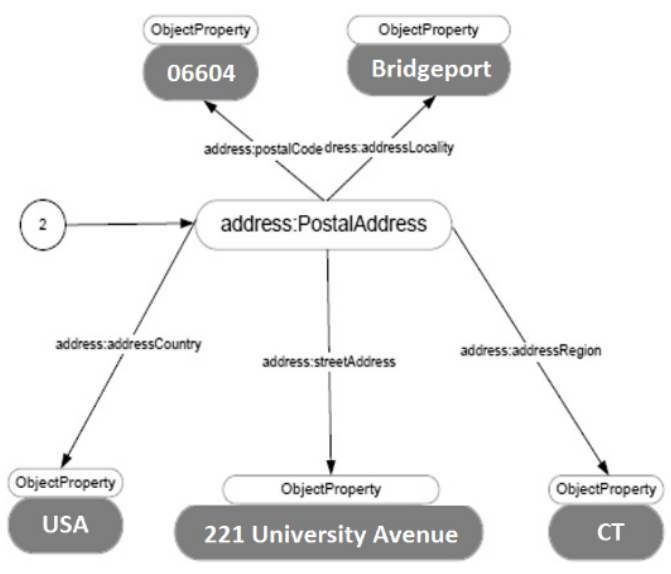

Fig. 14.Relations $1 / 4$

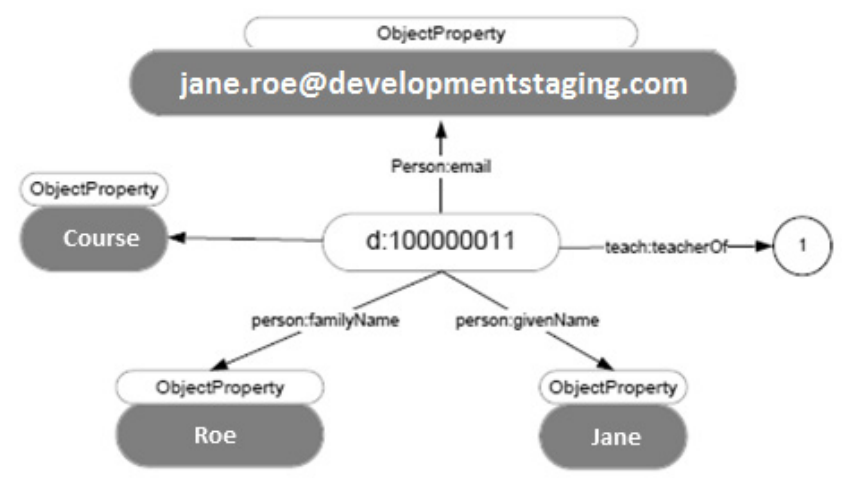

Fig. 15.Relations $2 / 4$ 
Paper-Updating Student Profiles in Adaptive Mobile Learning using ASP.net MVC, dotNetRDF,...

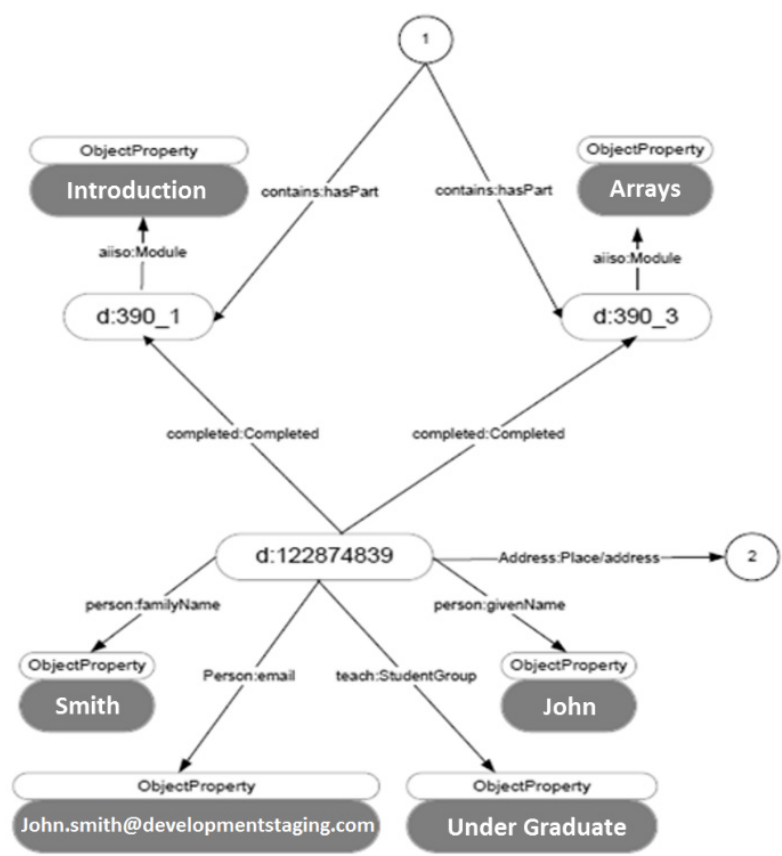

Fig. 16. Relations $3 / 4$

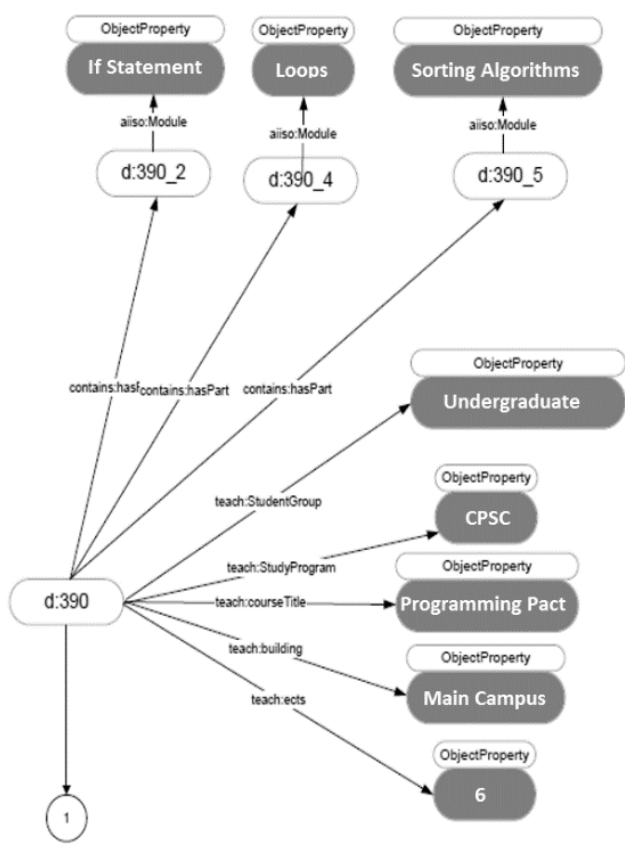

Fig. 17.Relations $4 / 4$ 
The following Turtle files were used to illustrate the relations on Figures 14 through 17:

1. CourseModules.ttl

2. Courses.ttl

3. Courses CourseModules.ttl

4. Faculty.ttl

5. FacultyCourses.ttl

6. Students.ttl

7. StudentsModules.ttl

As indicated from these figures, we have a student with the following attributes:

1. ID $=122874839$

2. Given Name ="John"

3. Family Name = "Smith"

4. Email="john.smit@developmentstaging.com"

5. Student Group = "Under Graduate"

6. Postal Address:

7. Street Address "221 University Avenue"

8. Address Locality = "Bridgeport"

9. Address Region $=$ "CT"

10. Postal Code = "06604"

11. Country = "USA"

John Smith has completed the following course units:

1. Introduction

2. Arrays

The Introduction and Arrays are parts of the course 390, which has the following attributes:

1. Study Program = "CPSC"

2. Course Title $=$ "Programming Pact"

3. Building = "Main Campus"

4. ects "Credits" = "6"

5. Student Group = "Undergraduate"

This course has five modules:

1. "Introduction"

2. "If Statement"

3. "Arrays"

4. "Loops"

5. "Sorting Algorithms"

This course has an instructor with the following attributes: 
Given Name $=$ "Jane"

Family Name = "Roe";

Email="jane.roe@developmentstaging.com"

Teacher $=$ "course"

We could, also, query that the grade is in a specific acceptable range, as described in the Semantic Web: Real World Applications from Industry Book to using RUD (University Resource Descriptor) [20], SUD (Student University Descriptor), RQL (RDF Query Language), RDQL (RDF Data Query Language), SWRL (Semantic Web Rule Language), Buchingae, SPARQL, as shown in Figure 18.

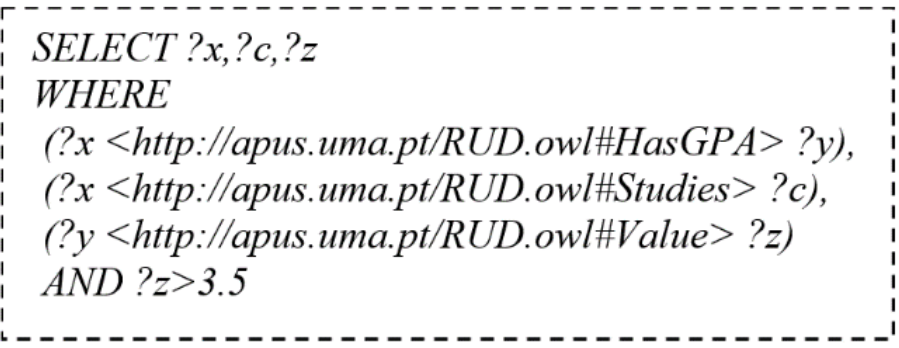

Fig. 18.Querying Students with GPA > 3.5

\section{Experiment}

In this section, we present the results of our experiment. For this experiment, we have used the Network Security course CPEG 561. CPEG 561 is a graduate course offered as an elective for Computer Science and Computer Engineering students. We tested two student groups, the AML Group and the Control Group. The AML is the group that used our system. Both the AML group and the Control Group completed a Pre-Quiz. The Pre-Quiz included 25 multiple choice questions and true/false questions. The Post-Quizzes contained three essay questions and the other 5 quizzes contained 66 multiple choice and true/false questions, as follows:

Quiz $1 \rightarrow 16$ Questions

Quiz $2 \rightarrow 12$ Questions

Quiz $3 \rightarrow 12$ Questions

Quiz $4 \rightarrow 12$ Questions

Quiz $5 \rightarrow 14$ Questions

Table 4 shows the grades of the AML group and the Control Group for the PreQuiz and Post Quizzes 
Paper-Updating Student Profiles in Adaptive Mobile Learning using ASP.net MVC, dotNetRDF,...

Table 4. Students Grades in our Study

\begin{tabular}{|c|c|}
\hline \multicolumn{2}{|c|}{ AML Group } \\
\hline Pre-Quiz & Post-Quizzes \\
\hline 56.00 & 50.12 \\
\hline 80.00 & 53.89 \\
\hline 56.00 & 55.10 \\
\hline 60.00 & 57.11 \\
\hline 56.00 & 61.47 \\
\hline 64.00 & 63.91 \\
\hline 52.00 & 65.98 \\
\hline 60.00 & 67.27 \\
\hline 84.00 & 69.13 \\
\hline 56.00 & 70.47 \\
\hline 60.00 & 71.45 \\
\hline 36.00 & 74.44 \\
\hline 56.00 & 74.76 \\
\hline 44.00 & 77.92 \\
\hline 48.00 & 85.35 \\
\hline
\end{tabular}

\begin{tabular}{|c|c|}
\hline \multicolumn{2}{|c|}{ Control Group } \\
\hline Pre-Quiz & Post-Quizzes \\
\hline 72.00 & 1.56 \\
\hline 40.00 & 37.72 \\
\hline 48.00 & 41.33 \\
\hline 84.00 & 42.78 \\
\hline 80.00 & 42.82 \\
\hline 56.00 & 43.90 \\
\hline 64.00 & 44.37 \\
\hline 64.00 & 44.54 \\
\hline 52.00 & 47.40 \\
\hline 84.00 & 47.98 \\
\hline 84.00 & 49.96 \\
\hline 72.00 & 52.40 \\
\hline 72.00 & 55.58 \\
\hline 76.00 & 65.38 \\
\hline 60.00 & 71.84 \\
\hline
\end{tabular}

Figures 19 and 20, illustrate the grades of both Groups for the Pre-quiz and the Post-Quizzes. The graphs clearly show improvement in the AML Group compared to the Control Group.

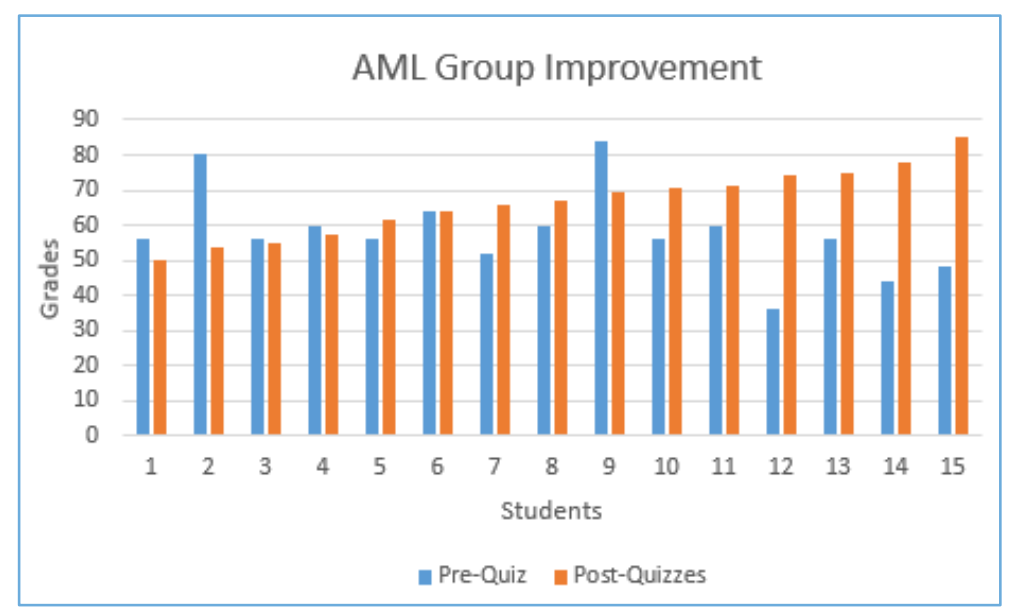

Fig. 19.AML Group Improvement 


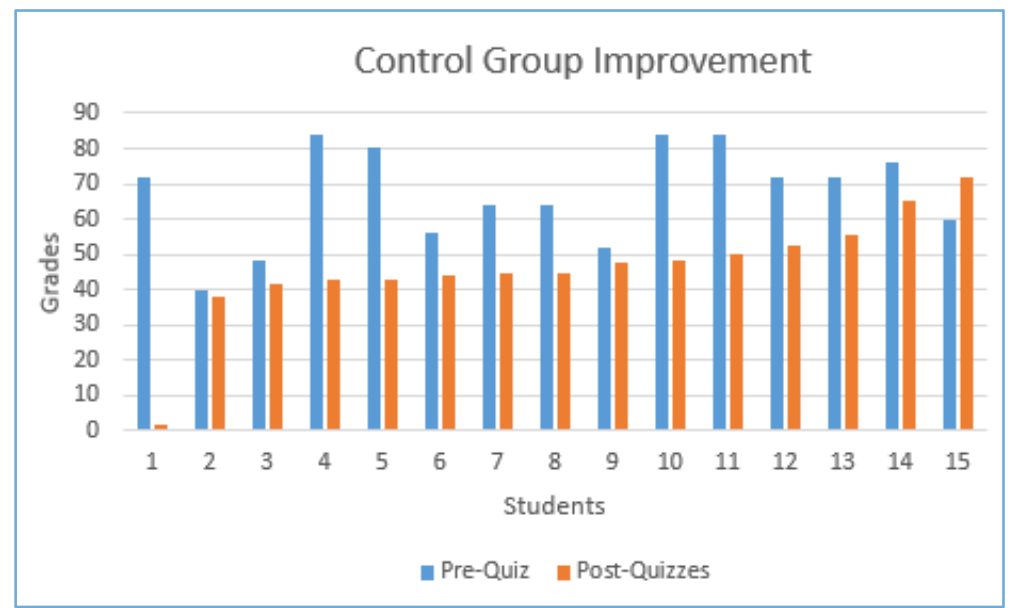

Fig. 20.Control Group Improvement

Figure 21 illustrates the distribution of the grades in the two groups.

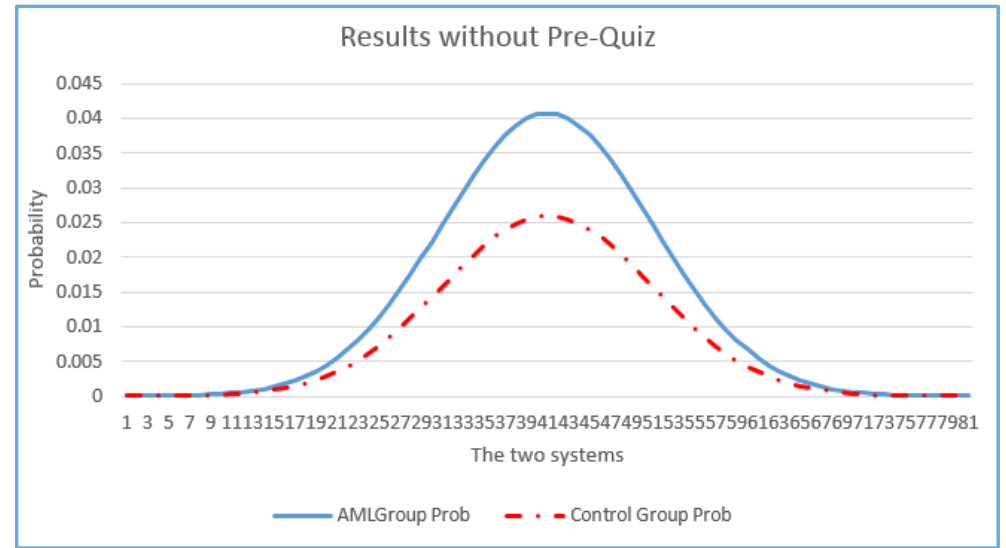

Fig. 21.The distribution of the grades in the two groups

AML Group Average for Post-Quizzes $=66.56$

Control Group Average for Post-Quizzes = 45.97

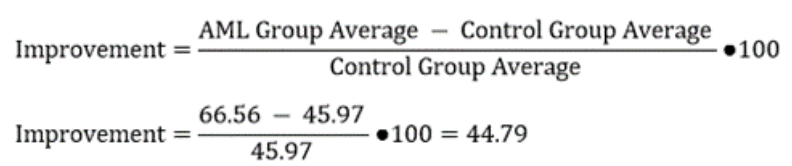

From the previous tables and charts, we can see that the AML Group is more effective $(44.79 \%)$ than the Control Group, furthermore, the grades in the AML group are 
steadier than the Control Group. A statistical analysis is conducted, to demonstrate that the results are statistically significant.

The parameters below are computed in order to decide whether to accept or reject the hypotheses $\mathrm{H}_{0}$.

\section{Hypothesis Testing}

- $\quad$ Null hypothesis $\left(\mathrm{H}_{0}\right)$ :

$$
\bar{X}_{A M L} \leq \bar{X}_{\text {Control Group }}
$$

These two groups have the same outcome.

- $\quad$ Alternative hypothesis $\left(\mathrm{H}_{\mathrm{a}}\right)$ :

$$
\bar{X}_{A M L}>\bar{X}_{\text {Control Group }}
$$

These two groups do not have the same outcome.

Where:

$\bar{X}_{A M L}$ is the AML Group mean.

$\bar{X}_{\text {Control Group }}$ is the Control Group mean.

By doing two tails t-value calculations.

The calculated $\mathrm{t}$-value $=4.38$

The Critical t-value (with $\alpha=0.05)=2.05$

According to the values above, the calculated t-value is greater than the Critical tvalue. We reject the Null Hypothesis, because the calculated t-value of 4.38 is larger than the Critical value which is 2.05 with $95 \%$ confidence level. This proves that the results are statistically significant.

\section{Conclusions}

On March 11, 2016, prior to any survey data were collected, an Institutional Review Board (IRB) approval was granted at the University of Bridgeport. The AML system indicated substantial improvement over the Control System. The experiment has shown that we could use the Semantic Web with adaptive mobile learning to enhance the courses, making them more dynamic. The Semantic Web obtains the information about completed course units, which are applied to the learning path graph, and a new optimal path is generated. Furthermore, if the student completes the target module, the student does not have to go through the rest of the modules. We can also prevent teaching the same course unit for the student more than once. Thus, the proposed approach could significantly improve the cost effectiveness for the stu- 
dents causing them to better manage their time more efficiently. Since there is a preview component to each course, we made sure that the students have the required information. The approach presented in this paper is expected to improve the performance of adaptive mobile learning and provide a learning experience to students that is more personalized and dynamic.

\section{$7 \quad$ References}

[1] (30 Jan. 2016). What is eLearning? Available: http://www.elearningnc.gov/about_ele arning/what_is_elearning/

[2] W3.org. (25 Oct. 2015). SPARQL 1.1 Protocol. Available: http://www.w3.org/TR/ sparq111-protocol/

[3] (2016). RDF Current Status Available: https://www.w3.org/standards/techs/rdf\#w3c all

[4] (2016). Turtle - Terse RDF Triple Language. Available: https://www.w3.org/ TeamSubmission/turtle/

[5] C. S. Bhatia and S. Jain, "Semantic Web Mining: Using Ontology Learning and Grammatical Rule Inference Technique," in 2011 International Conference on Process Automation, Control and Computing (PACC), 2011, pp. 1-6. https://doi.org/10.1109/PA CC.2011.5979024

[6] A. S. Hadi, P. Fergus, C. Dobbins, and A. M. Al-Bakry, "A Machine Learning Algorithm for Searching Vectorised RDF Data," in 2013 27th International Conference on Advanced Information Networking and Applications Workshops (WAINA), 2013, pp. 613-618. https://doi.org/10.1109/waina.2013.204

[7] F. Soualah-Alila, F. Mendes, and C. Nicolle, "A Context-Based Adaptation In Mobile Learning," IEEE Computer Society Technical Committee on Learning Technology (TCLT), vol. 15, p. 5 pages, 2013.

[8] F. Grivokostopoulou, I. Perikos, and I. Hatzilygeroudis, "Utilizing semantic web technologies and data mining techniques to analyze students learning and predict final performance," in 2014 International Conference on Teaching, Assessment and Learning (TALE), 2014, pp. 488-494. https://doi.org/10.1109/TALE.2014.7062571

[9] A. Walia, N. Singhal, and A. K. Sharma, "A Novel E-learning Approach to Add More Cognition to Semantic Web," in 2015 IEEE International Conference on Computational Intelligence \& Communication Technology (CICT), 2015, pp. 13-17. https://doi.org/10.1109/cict.2015.15

[10] L. Kagal, T. Finin, and A. Joshi, "A policy based approach to security for the semantic web," in International Semantic Web Conference, 2003, pp. 402-418. https://doi.org/10.1007/978-3-540-39718-2 26

[11] B. Thuraisingham, "Security issues for the semantic Web," in Computer Software and Applications Conference, 2003. COMPSAC 2003. Proceedings. 27th Annual International, 2003, pp. 633-638. https://doi.org/10.1109/cmpsac.2003.1245408

[12] I. A. Alshalabi, S. Hamada, and K. Elleithy, "Automated adaptive learning using smart shortest path algorithm for course units," in 2015 IEEE Long Island Systems, Applications and Technology Conference (LISAT 2015), 2015, pp. 1-5. https://doi.org/10.1109/lisat.2015.7160187

[13] S. Hamada, I. A. Alshalabi, K. Elleithy, and I. Badara, "Automated Adaptive Mobile Learning System using the Semantic WEB," in 2016 IEEE Long Island Systems, Applications and Technology Conference (LISAT 2016), 2016. https://doi.org/10.1109/ lisat.2016.7494150 
[14] B. DuCharme, Learning Sparql: O'Reilly Media, Inc., 2013.

[15] J. H. Sofia, B. D. Sofia, A. D. José, and J. H. Leontios, Fuzzy Logic-Based Modeling in Collaborative and Blended Learning. Hershey, PA, USA: IGI Global, 2015.

[16] M. Eisenstadt and T. Vincent, The knowledge web: Learning and collaborating on the net: Routledge, 2012.

[17] L. Ding, P. Kolari, Z. Ding, and S. Avancha, "Using ontologies in the semantic web: A survey," in Ontologies, ed: Springer, 2007, pp. 79-113. https://doi.org/10.1007/978-0-387$37022-4 \quad 4$

[18] J. Gajek. (28 Oct. 2015). Ontology, Notation 3, and SPARQL. Available: http://www.codeproject.com/Articles/156888/Ontology-Notation-and-SPARQL

[19] (14 Apr. 2016). dotNetRDF - Semantic Web, RDF and SPARQL Library for C\#/.Net. Available: http://dotnetrdf.org/

[20] J. Cardoso, "Developing course management systems using the Semantic Web," in The Semantic Web. vol. Chapter 10, ed: Springer, 2008, pp. 169-188. https://doi.org/10.1007/ 978-0-387-48531-7 8

\section{Authors}

Dr. Samir Hamada received his $\mathrm{PhD}$ in Computer Science and Engineering and MS in Computer Science from University of Bridgeport in 2017 and 2001. He, also, received his B.S. in Accounting from Ain Shams University in Egypt. He is currently an Assistant Professor of Computer Systems, School of Business at Farmingdale State College in Farmingdale NY, USA. His research interests include Adaptive Learning, Mobile Learning and the Semantic Web. (e-mail: shamada@bridgeport.edu)

Dr Ibrahim Alkore Alshalabi received his B.Sc. in Computer Science from AlIsra Private University, Amman, Jordan in 1997, his Master of Computer Applications (MCA) from Bangalore University, India in 2007, and his $\mathrm{PhD}$ in Computer Science and Engineering from the University of Bridgeport, USA in 2016. From 1997-2004 he was Assistant Lecturer at Ma'an Community College, Al-Balqa Applied University, Jordan. From 2007 to 2009 he was an assistant lecturer at AlHussein Bin Talal University, Jordan. He is currently an adjunct Professor at AlHussein Bin Talal University, College of Information technology, Jordan. His research interests are E-Learning, M-Learning, wireless communications, and networks. He was an active committee member of the International Conference on Engineering Education, instructional technology, Assessment, and E-Learning (EIAE 2010, EIAE 2011). (e-mail: ialkorea@my.bridgeport.edu)

Dr. Khaled Elleithy is the Associate Vice President for Graduate Studies and Research at the University of Bridgeport. He is a professor of Computer Science and Engineering. His research interests include wireless sensor networks, mobile communications, network security, quantum computing, and formal approaches for design and verification. He has published more than three hundred fifty research papers in national / international journals and conferences in his areas of expertise. Dr. Elleithy is the editor or co-editor for 12 books published by Springer.

Dr. Elleithy received the B.Sc. degree in computer science and automatic control from Alexandria University in 1983, the MS Degree in computer networks from the same university in 1986, and the MS and Ph.D. degrees in computer science from The 
Center for Advanced Computer Studies at the University of Louisiana - Lafayette in 1988 and 1990, respectively.

Prof. Elleithy has more than 30 years of teaching experience. His teaching evaluations were distinguished in all the universities he joined. He is the recipient of the "Distinguished Professor of the Year", University of Bridgeport, academic year 20062007. He supervised hundreds of senior projects, MS theses and Ph.D. dissertations. He developed and introduced many new undergraduate/graduate courses. He also developed new teaching / research laboratories in his area of expertise. His students have won more than twenty prestigious national / international awards from IEEE, ACM, and ASEE.

Dr. Elleithy is a member of the technical program committees of many international conferences as recognition of his research qualifications. He served as a guest editor for several international journals. He was the chairperson of the International Conference on Industrial Electronics, Technology \& Automation. Furthermore, he is the co-Chair and co-founder of the Annual International Joint Conferences on Computer, Information, and Systems Sciences, and Engineering virtual conferences 2005 - 2014.

Dr. Elleithy is a member of several technical and honorary societies. He is a Senior Member of the IEEE computer society. He is a member of the Association of Computing Machinery (ACM) since 1990, member of ACM SIGARCH (Special Interest Group on Computer Architecture) since 1990, member of the honor society of Phi Kappa Phi University of South Western Louisiana Chapter since April 1989, member of IEEE Circuits \& Systems society since 1988, member of the IEEE Computer Society since 1988, and a lifetime member of the Egyptian Engineering Syndicate since June 1983. (e-mail: elleithy@bridgeport.edu)

Dr. Ioana Badara holds a Ph.D. (Teacher Preparation/Science Education) from University of Tennessee-Knoxville and an M.Phil. (Microbiology \& Immunology) from University of Edinburgh, Scotland. Prior to completing her doctoral work, she has worked as a research scientist in the biomedical field for about ten years, having been affiliated with Weill Medical College of Cornell University and Mount Sinai School of Medicine, in New York City. Her passionate interest in the exploration of connections between scientists' epistemologies and the teaching of science led her to pursuing doctoral studies in Science Education. She has taught a multitude of core Biology courses for Biology/Pre-Medical undergraduates and mentored student research projects in this field. Dr. Badara is currently a faculty member at University of Bridgeport, where she teaches core research courses in the doctoral (Ed.D.) program, Science Education courses in the Science Teacher Preparation program, and History and Philosophy of Science courses at the undergraduate level. She has been the recipient of several grants for research, including a National Science Foundation grant for conducting research on the teaching of science in urban school districts. She has presented her work at national and international conferences in the field of STEM education. (e-mail: ibadara@bridgeport.edu)

Dr. Saeid Moslehpour is a Professor and Assistant Dean of Graduate Studies in College of Engineering, Technology, and Architecture at the University of Hartford. Currently he is currently editor of Computer in Education Journal of ASEE. He holds Ph.D. (1993) from Iowa State University, Master of Science (1990) and Bachelor of 
Science from University of Central Missouri and (1989). His research interests include modeling, simulation, CPLDs, FPGAs, analog/digital mixed, embedded electronic system testing, rapid prototyping and cyber learning. He is former faculty senate chair, ASEE Section I chair, co-director of Connecticut NASA Space Grant and ECE department chair. (e-mail: moslehpou@hartford.edu)

Article submitted 21 August 2016. Published as resubmitted by the authors 23 December 2016. 